12,05

\title{
Эффективная скорость движения доменной границы в квазиодномерной наносистеме при множественном рождении доменов новой фазы
}

\author{
(C) Б.В. Петухов \\ Институт кристаллографии им. А.В. Шубникова ФНИЦ „Кристаллография и фотоника“ РАН, \\ Москва, Россия \\ E-mail: petukhov@ns.crys.ras.ru
}

(Поступила в Редакцию 12 декабря 2017 г.)

\begin{abstract}
Процесс переключения метастабильного состояния в энергетически предпочтительное в протяженных квазиодномерных системах нередко происходит посредством движения границ доменов новой фазы. Обычно рассматриваемые предельные случаи - это распространение на всю систему краевого домена или множественное зарождение и коалесценция доменов в объеме материала. В работе рассматривается более общая ситуация распространения фронта переключения состояния от границы образца при множественном рождении доменов новой фазы на случайно расположенных в объеме дефектах. Решается соответствующая статистико-кинетическая задача с расчетом средней величины и дисперсии длин пробегов, а также полной производящей функции для нахождения высших моментов распределения длин „эстафетных“ пробегов границы краевого домена.
\end{abstract}

DOI: $10.21883 /$ FTT.2018.06.46007.347

\section{1. Введение}

Движение фронта изменения состояния является важной составляющей процессов перестройки систем самой различной природы. Наиболее изученными случаями являются движение доменных стенок при фазовых превращениях в металлах и сплавах [1] и в магнитных материалах [2]. В качестве нескольких других показательных примеров можно упомянуть процессы кристаллизации $[3,4]$, используемые в так называемых CMOS технологиях переходы кристаллическое-аморфное состояние в силицидах $[5,6]$, денатурация и репликация молекул ДНК $[7,8]$, движение дислокационных кинков [9] и т.п. Типичный представитель семейства квазодномерных наносистем - ферромагнитные нанопроволоки - в недавнее время привлекли значительный интерес в связи с возможными приложениями в устройствах хранения информации [10-13]. Благодаря малому диаметру нанопроволок предполагается, что их использование приведет к повышению плотности записи информации. Перемагничивание микро- и нанопроволок при изменении направления магнитного поля происходит посредством образования доменов локально переориентированного состояния и их распространения на всю систему за счет движения доменных границ. Изучению этого процесса и возможности управлять его кинетикой посвящены многочисленные работы [14-19] и др.

В работе [20] механизмом повышения скорости переключения состояния намагниченности микропроволоки предложено считать зарождение новых доменов перед движущейся исходной доменной стенкой на активных центрах, создаваемых дефектами материала. Одна из границ нового домена движется навстречу исходной доменной стенке, и в результате слияния доменов фронт переключения скачком переходит в положение второй границы нового домена. При множественном образовании доменов такой процесс многократно повторяется. Это отвечает эффективному увеличению подвижности фронта переключения состояния, поскольку движение фронта в подобных условиях определяется не только скоростью перемещения одной доменной границы, а суммарным движением многих границ. Расчет характеристик эффективной подвижности фронта переключения состояния при множественном зарождении доменов новой фазы на хаотически расположенных дефектах материала и является целью настоящей работы.

\section{2. Свойства активной среды, образованной зарождением доменов новой фазы на дефектах материала}

Согласно многим наблюдениям, новые домены возникают на хаотически распределенных дефектах материала, как это проиллюстрировано на рис. 1, показывающем схему эксперимента по измерению скорости перемещения границы краевого домена. Кинетика зарождения новой фазы на каких-то активных центрах типа примесей и т.п., изучалась в [4,9,21,22]. Рассматривается ситуация, когда величина движущей силы процесса недостаточна для снятия барьера между состояниями, так что исходное состояние сохраняет метастабильную устойчивость. Но все же она достаточно велика, чтобы нуклеация, то есть флуктуационное образование зародышей новой фазы в исходной среде занимало малый 


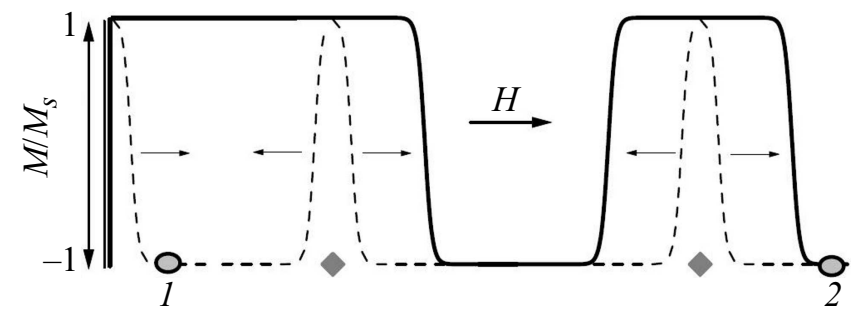

Рис. 1. Схема эксперимента по исследованию движения доменной границы, введенной на левом конце образца, между измерительными катушками, расположенными в позициях 1 и $2 . H$ - переключающее поле, $M-$ намагниченность, $M_{s}-$ намагниченность насыщения. Символы обозначают активные центры, на которых зарождаются домены новой фазы. Штриховые линии показывают начальное состояние, сплошные линии показывают конфигурацию доменов спустя какое-то время с учетом поглощения фронтом переключения состояния домена новой фазы, образованного на ближайшем активном центре.

так называемый критический размер и происходило независимо друг от друга. Дальнейший рост зародышей может описываться на языке движения границ доменов с определенной дрейфовой скоростью $v$. Скорость движения границ доменов $v$ при постоянной внешней движущей силе будет приниматься постоянной. При сближении соседних доменов их границы аннигилируют и пара доменов превращается в один большего размеpa. В случае, когда средняя длина пробега доменных границ до взаимной аннигиляции много больше критического размера зародышей, последний можно считать нулевым.

Фронт переключения состояния, стартующий от края системы, движется через неупорядоченную активную среду, образованную чередой доменов новой фазы, растущих от хаотически расположенных дефектов материала и слипающихся с краевым доменом. Опишем вкратце некоторые известные количественные характеристики этой среды вдали от границ образца и, в первую очередь, приведем распределение по размерам убывающих со временем промежутков между доменами. Обозначим плотность активных центров на единице длины системы как I. Вероятность того, что с какойлибо стороны, например, справа от данной точки, интервал длиной, превышающей $l_{1}$, не будет захвачен до времени $t$ родившимся с этой стороны доменом новой фазы, может быть записана как вероятность отсутствия активного центра на длине $l_{1}+v t$, которая при хаотическом расположении центров равна $\exp \left[-\left(l_{1}+v t\right) I\right]$. Добавление $v t$ к $l_{1}$ гарантирует, что граница интервала не будет до времени $t$ нарушена доменом, родившимся в пределах преодолеваемого за время $t$ расстояния $v t$ от границы.

Дифференцируя ехр $\left[-\left(l_{1}+v t\right) I\right]$ по $l_{1}$, получаем вероятность иметь справа свободный интервал длиной от $l_{1}$ до $l_{1}+d l_{1}$ в виде $I \exp \left[-\left(l_{1}+v t\right) I\right] d l_{1}$. Аналогично и слева для интервала между $l_{2}$ и $l_{2}+d l_{2}$. Плотность ве- роятности $p_{1}(l, t)$ данной точке принадлежать интервалу длиной от $l$ до $l+d l$ для любых $l_{1}$ и $l_{2}$ есть

$$
\begin{aligned}
& p_{1}(l, t)=\int_{0}^{\infty} d l_{1} \int_{0}^{\infty} d l_{2} \delta\left(l_{1}+l_{2}-l\right) I^{2} \\
& \quad \times \exp \left[-I\left(l_{1}+l_{2}+2 v t\right)\right]=I^{2} l \exp [-I(l+2 v t)] .
\end{aligned}
$$

Отметим, что вероятность данной точке принадлежать хоть какому-то свободному интервалу равна интегралу от $p_{1}(l, t)$ по всем $l$, что дает, как нетрудно видеть, $\exp (-2 v t I)$, что есть просто доля исходной фазы, как и должно быть.

Далее понадобится также плотность распределения свободных промежутков между доменами новой фазы данного размера в другой нормировке - в расчете на единицу длины системы. В работах [23-26] для решения подобной задачи применялось кинетическое уравнение, здесь же, следуя методу работ [27,28], будет показано, как нужная величина может быть найдена с помощью полученной выше плотности вероятности данной точке принадлежать интервалу длиной от $l$ до $l+d l \quad p_{1}(l, t)=I^{2} l \exp [-I(l+2 v t)]$ более простым путем. Разобьем интервал $l$ на ячейки малой длины $\Delta l$. Их число в данном интервале примерно равно $n \approx l / \Delta l$. Вероятность данной ячейке принадлежать свободному интервалу из $n$ ячеек есть $p_{1}(l, t) \Delta l$. Пусть длина системы есть $L \approx N \Delta l$. Подсчитаем полное число на ней свободных интервалов из $n$ ячеек. Если умножить вероятность $p_{1}(l, t) \Delta l$ на $N$, то каждый интервал будет в $N p_{1}(l, t) \Delta l$ учитываться столько раз, сколько в нем ячеек. Поэтому число разных интервалов получается делением $N p_{1}(l, t) \Delta l$ на $n$ и равно $N_{l}=(N / n) p_{1}(l, t) \Delta l$. Чтобы найти число таких интервалов на единице длины системы, поделим $N_{l}$ на $L$. Имеем в результате $I^{2} \exp [-I(l+2 v t)] \Delta l$, то есть, возвращаясь к континуальному описанию, находим вероятность встретить свободный интервал длиной от $l$ до $l+d l$ в расчете на единицу длины равной

$$
p^{\prime}(l, t) d l=I^{2} \exp [-I(l+2 v t)] d l .
$$

Полная плотность непереключенных интервалов на единицу длины $N(t)$ равна интегралу от $p^{\prime}(l, t)$ по всем $l$, то есть $N(t)=I \exp (-2 I v t)$. Деля $p^{\prime}(l, t)$ на $N(t)$, получаем вероятности встретить непереключенный интервал длиной $l$ в виде $p(l, t)=I \exp (-I l)$

Расчет характеристик доменов новой фазы представляет собой более сложную задачу, рассматриваемую в работах [24-26]. Однако некоторые из этих характеристик могут быть найдены элементарно, в частности, эволюция со временем плотности доменов новой фазы и их средних длин. Так как непереключенные интервалы чередуются с переключенными, рассчитанная выше величина $N(t)=I \exp (-2 I v t)$ представляет собой также и плотность доменов новой фазы. Средняя длина непереключенных интервалов есть $\left\langle l_{-}\right\rangle=\int_{0}^{\infty} l p(l, t) d l=1 / I$. 
Среднее расстояние между доменами одного типа равно $1 / N(t)=(1 / I) \exp (2 v I t)$. Эта величина складывается из средней длины непереключенных интервалов $\left\langle l_{-}\right\rangle=1 / I$ и среднего размера домена новой фазы $\left\langle l_{+}\right\rangle$, таким образом,

$$
\left\langle l_{+}\right\rangle=1 / N(t)-\left\langle l_{-}\right\rangle=(1 / I)[\exp (2 v I t)-1] .
$$

\section{3. Статистическое описание продвижения фронта переключения состояния}

Ввиду случайного расположения центров зарождения доменов новой фазы перемещение фронта переключения с учетом коалесценции доменов представляет собой стохастический процесс и должен, вообще говоря, характеризоваться некоторой функцией распределения $p_{b}(x, t)$ - вероятностью нахождения границы в момент $t$ в интервале между $x$ и $x+d x$. В имеющихся публикациях большое внимание уделялось расчетам кинетики размеров доменов новой фазы в неограниченной среде на единицу длины системы, характеризуемой некоторой плотностью вероятности $g(x, t)$ [24-26]. Это не вполне та величина, которая нам нужна $-p_{b}(x, t)$ относится к полубесконечной системе - однако с ней имеется довольно простая связь, что позволит использовать результаты, полученные ранее.

Кинетическое уравнение для $p_{b}(x, t)$ записывается аналогично уравнению для $g(x, t)$ работы [24] и имеет вид

$$
\begin{aligned}
\frac{\partial p_{b}(x, t)}{\partial t}= & -v \frac{\partial p_{b}(x, t)}{\partial x}-v I p_{b}(x, t) \\
& +v \gamma \int_{0}^{x} g\left(x-x^{\prime}, t\right) p_{b}\left(x^{\prime}, t\right) d x^{\prime} .
\end{aligned}
$$

Здесь $\gamma=\exp (2 v I t)$. Первое слагаемое в правой части (4) описывает расширение краевого домена, второе слагаемое представляет уход домена данного размера в большие размеры из-за столкновений с объемными доменами, интеграл описывает образование домена данного размера вследствие коалесценции краевого и объемного доменов меньших размеров. Начальное условие есть $p_{b}(x, t=0)=\delta(x)$. Очевидно также, что $p_{b}(x, t)=0$, если $x$ меньше детерминированного предела $v t$ распространения краевого домена.

В лапласовом представлении уравнение (4) принимает вид

$$
\frac{\partial p_{b}(s, t)}{\partial t}=-v[s+I-\gamma g(s, t)] p_{b}(s, t) .
$$

При известном из работы [25] лапласовом образе $g(x, t)=(I+s) /\left[\gamma^{2}(s / I) \exp (2 s v t)+\gamma\right]$ решение уравне-

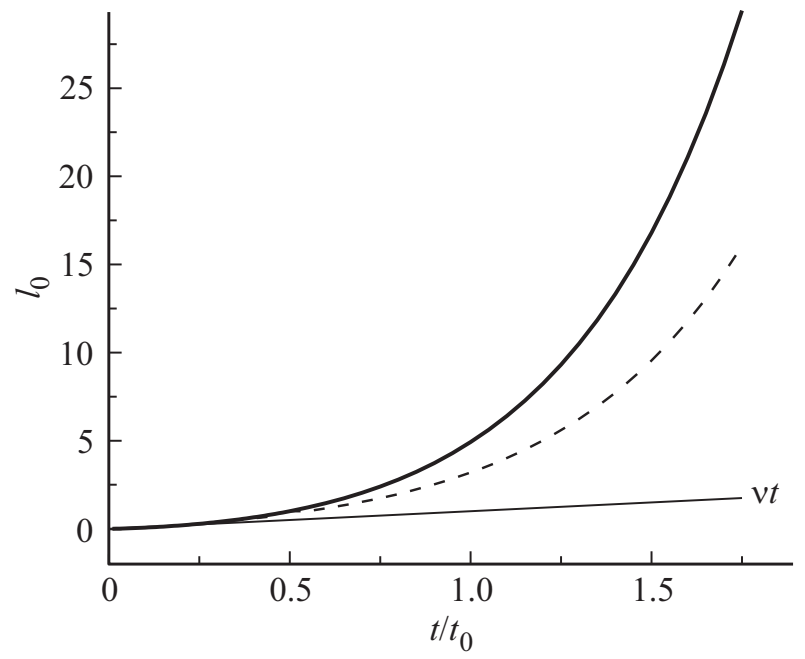

Рис. 2. Изменение со временем характерного масштаба пробегов фронта переключения состояния (в единицах $1 / I$, жирная линия). Штриховая линия изображает временную зависимость средней длины пробега. Тонкая линия соответствует движению границы краевого домена с постоянной скоростью $v$.

ния (5) есть

$p_{b}(s, t)=K(s) \exp \left\{-v(s+I) t+v \int^{t} d t^{\prime} g\left(s, t^{\prime}\right) \exp \left(2 I v t^{\prime}\right)\right\}$,

где $K(s)$ - функция, определяемая начальным условием $p_{b}(s, t=0)=1$. После интегрирования и применения начального условия получаем

$$
p_{b}(s, t)=\left[\frac{1+s / I}{(\gamma s / I) \exp (2 s v t)+1}\right]^{1 / 2} .
$$

Лапласов образ $p_{b}(s, t)$ не допускает простого аналитического обращения, однако он позволяет получить некоторые полезные характеристики оригинала $p_{b}(x, t)$, например, исследовать закон убывания больших длин пробегов. Он определяется сингулярностями $p_{b}(s, t)$, обусловленными обращением в нуль знаменателя в (7) $p_{b}(x, t) \sim \exp \left(s_{0} x\right)$, где $s_{0}$ есть корень уравнения

$$
s \exp (2 s v t)=-I / \gamma .
$$

Один корень $s=-I$ не дает вклада в $p_{b}(x, t)$ из-за обращения в нуль числителя в (7). Подходящим решением является

$$
s_{0}=\frac{1}{2 v t} W[-2 I v t \exp (-2 I v t)]
$$

где $W(z)$ есть функция Ламберта [29]. Определяемый (9) характерный масштаб спадания вероятности больших длин пробегов $l_{0}=-1 / s_{0}$ изображен на рис. 2 в зависимости от времени. Как можно видеть, вероятность больших длин пробегов фронта переключения состояния резко нарастает со временем благодаря включению в игру коалесценции с объемными доменами. 


\section{4. Вычисление средних величин}

Лапласов образ $p_{b}(s, t)$ является производящей функцией, позволяющей находить различные моменты распределения длин пробегов фронта переключения состояния. Средняя длина пробега выражается через $p_{b}(s, t)$ как

$$
\begin{aligned}
\left\langle l_{b}(t)\right\rangle & =\int_{0}^{\infty} d x x p_{b}(x, t) \\
& =-\left.\frac{\partial p_{b}(s, t)}{\partial s}\right|_{s=0}=\frac{1}{2 I}[\exp (2 I v t)-1] .
\end{aligned}
$$

Полученная величина составляет половину среднего размера домена нового состояния (3), как и следовало ожидать с учетом того обстоятельства, что рост краевого домена происходит лишь в одну сторону. Эта функция имеет пределы $\left\langle l_{b}\right\rangle \approx v t, t \rightarrow 0,\left\langle l_{b}\right\rangle \sim \frac{1}{2 I} \exp (2 v I t)$, $t \rightarrow \infty$, то есть при малых временах граница движется свободно с индивидуальной скоростью $v$, а при больших временах перемещение фронта переключения в основном определяется слияниями с другими доменами, и эффективная скорость стенки резко нарастает (см. рис. 3). В этом случае, как показывает расчет кинетических кривых методом клеточных автоматов для конкретных случайных расположений дефектов, служащих центрами зарождения новой фазы, имеет место высокая степень стохастичности процесса. Плавное нарастание пробега перемежается со скачкообразным увеличением в случайные моменты столкновений фронта с доменами в объеме материала.

Средний квадрат пробега находится как

$$
\begin{aligned}
\left\langle l_{b}(t)^{2}\right\rangle & =\int_{0}^{\infty} d x x^{2} p_{b}(x, t) \\
& =\frac{\partial^{2} p_{b}(s, t)}{\partial s^{2}}=\frac{1}{2 I^{2}}\left(2 \gamma^{2}-4 \gamma I v t-\gamma\right) .
\end{aligned}
$$

Соответственно, дисперсия длин пробегов равна

$$
\left\langle\left[l_{b}(t)-\left\langle l_{b}(t)\right\rangle\right]^{2}\right\rangle=\frac{1}{2 I^{2}}\left(\gamma^{2}-4 \gamma I v t-1\right) .
$$

Аналогично могут быть получены и последующие моменты функции распределения

$$
\left\langle l_{b}(t)^{2}\right\rangle=(-1)^{n} \frac{\partial^{n} p_{b}(s, t)}{\partial s^{n}} .
$$

На рис. 4 изображена величина

$$
\begin{aligned}
f r & =\left(\left\langle l_{b}^{2}\right\rangle-\left\langle l_{b}\right\rangle^{2}\right)^{1 / 2} /\left\langle l_{b}\right\rangle \\
& =\sqrt{2}\left(\gamma^{2}-4 \gamma I \nu t-1\right)^{1 / 2} /(\gamma-1),
\end{aligned}
$$

представляющая собой отношение среднеквадратичного пробега к среднему пробегу и характеризующая флуктуации смещения фронта переключения состояния. Ее обращение в нуль при малых временах свидетельствует о

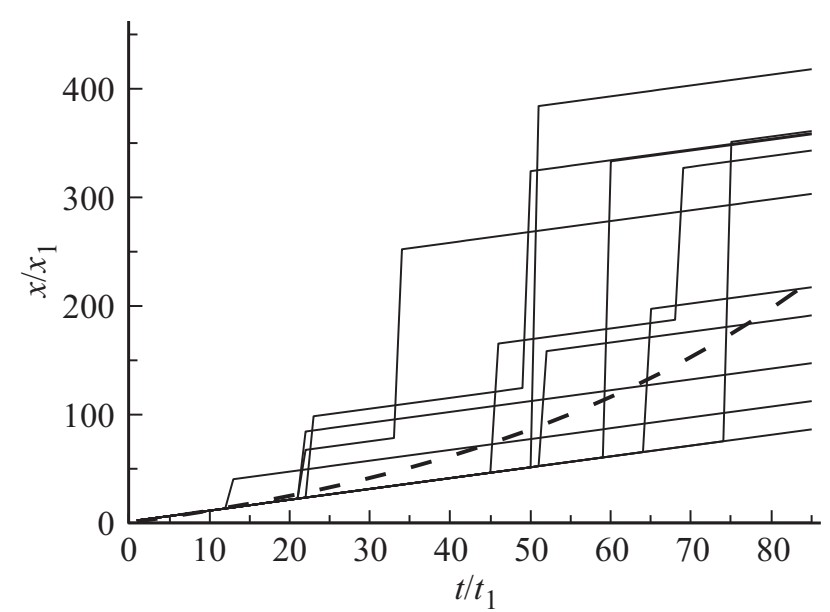

Рис. 3. Зависимость от времени длины пробега фронта переключения состояния. Ломаные линии изображают результат моделирования для различных конкретных случайных расположений дефектов, штриховая линия представляет среднюю длину пробега, даваемую формулой $(10),\left(x_{1}=0.01 / I\right.$, $\left.t_{1}=0.01 / v I\right)$.

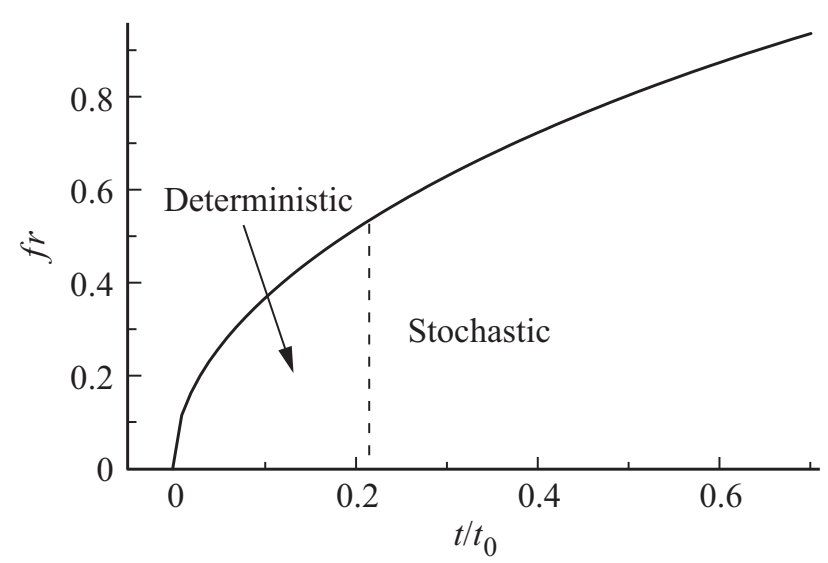

Рис. 4. Соотношение дисперсии и средней величины пробегов фронта переключения состояния $f r=\left(\left\langle l_{b}^{2}\right\rangle-\left\langle l_{b}\right\rangle^{2}\right)^{1 / 2} /\left\langle l_{b}\right\rangle$. Указана условная граница между детерминированным и стохастическими режимами движения фронта переключения состояния.

детерминистичности начальной кинетики фронта переключения, определяемой свободным движением границы краевого домена. Однако при больших временах порядка характерного времени системы $t_{0}=1 / \nu I$ размах флуктуаций пробега приближается к величине самого пробега, что показывает существенный вклад неупорядоченности системы и стохастичности взаимодействий с доменами новой фазы в кинетику переключения состояния.

\section{5. Заключение}

Проведенный расчет показал, что переключение состояний квазиодномерных систем значительно ускоряется при наличии активных центров зарождения дополнительных доменов новой фазы. Это может быть по- 
лезным в приложениях для повышения быстродействия наноустройств записи и хранения информации. Такой цели, как показывают эксперименты [19], могут служить определенные дефекты материала.

Качественное объяснение наблюдаемому повышению эффективной скорости переключения состояний с переходом к нелинейной зависимости подвижности от движущей силы может дать предложенный в работе [20] „тандемный“ механизм движения доменной стенки. Модельный вариант подобного механизма с передачей „эстафеты“ положения фронта переключения при столкновениях с доменами, образующимися в объеме материала, рассчитан в настоящей работе. Близкий по духу эффект влияния нелинейных возмущений, возникающих перед движущейся доменной границей и приводящий к возрастанию скорости перемещения фронта перемагничивания в полосках пермаллой-ниобий, изучался в работе [30]. Возможны, впрочем, и другие варианты, например, с изменением внутреннего устройства доменных стенок, если оно приводит к увеличению подвижности. В то же время наблюдаемая существенная стохастичность кинетики переключения состояний требует для надежной расшифровки лежащих в основе механизмов получения значительного объема статистических данных, чтобы сделать возможным количественное сопоставление с теоретическими моделями, принимающими во внимание неупорядоченность исследуемых материалов.

\section{Список литературы}

[1] Д. Кристиан. Теория превращений в металлах и сплавах. Мир, М. (1978). 806 с.

[2] В.В. Волков, В.А. Боков. ФТТ 50, 193 (2008).

[3] А.Н. Колмогоров. Изв. АН СССР. Сер. матем. 3, 355 (1937).

[4] В.З. Беленький. Геометрико-вероятностные модели кристаллизации. Наука, М. (1980). 88 с.

[5] S.-L. Zhang, \& Mikael Östling. Crit. Rev. Solid State Mater. Sci. 28, 1 (2010).

[6] A.L. Schmitt, J.M. Higgins, J.R. Szczech, S. Jin. J. Mater. Chem. 20, 223 (2010).

[7] D.K. Lubensky, D.R. Nelson. Phys. Rev. E 65, 031917 (2002).

[8] E.Z. Meilihov, E.M. Farzetdinova. Письма в ЖЭТФ 102, 60 (2015).

[9] Б.В. Петухов. Динамика дислокаций в кристаллическом рельефе. Дислокационные кинки и пластичность кристаллических материалов. Lambert Academic Publishing, Saarbrücken (2016). 385 c.

[10] Nanowires Science and Technology / Ed. N. Lupu. In-Tech (2010).

[11] D. Sellmyer, R. Skomski. Adv. Magn. Nanostructures. Springer (2006).

[12] One-Dimensional Nanostructures. (Ed) Z.M. Wang. Springer Science+Business Media, N.Y. (2008).

[13] Magnetic Nano- and Microwires. Design, Synthesis, Properties and Applications / Ed. M. Vázquez. Woodhead Publishing. Amsterdam (2015).

[14] T. Ono, H. Miyajima, K. Shigeto, K. Mibu, N. Hosoito, T. Shinjo. Science 284, 468 (1999).
[15] D. Atkinson, D.A. Allwood, G. Xiong, M.D. Cooke, C.C. Faulkner, R.P. Cowburn. Nature Mater. 2, 85 (2003).

[16] G.S.D. Beach, C. Nistor, C. Knutson, M. Tsoi, J.L. Erskine. Nature Mater. 4, 741 (2005).

[17] S.S.P. Parkin, M. Hayashi, L. Thomas. Science 320, 190 (2008).

[18] M. Vázquez, H. Chiriac, A. Zhukov, L. Panina, T. Uchiyama. Phys. Status Solidi A208, 493 (2011).

[19] R. Varga, A. Zhukov, V. Zhukova, J.M. Blanco, J. Gonzalez. Phys. Rev. B 76, 132406 (2007).

[20] M. Ipatov, V. Zhukova, A.K. Zvezdin, A. Zhukov. J. Appl. Phys. 106, 103902 (2009).

[21] M. Avrami. J. Chem. Phys. 7, 1103 (1939).

[22] Б.В. Петухов. Кристаллография 52, 113 (2007).

[23] K. Sekimoto. Physica A 125, 261 (1984).

[24] K. Sekimoto. Int. J. Mod. Phys. B 5, 1843 (1991).

[25] E. Ben-Naim, P.L. Krapivsky. Phys. Rev. E 54, 3562 (1996).

[26] S. Jun, H. Zhang, J. Bechhoefer. Phys. Rev. E 71, 011908 (2005).

[27] B. Petukhov. J. Stat. Mech. P09019 (2013).

[28] Б.В. Петухов. ФТП 47, 613 (2013).

[29] F.W.J. Olver, D.W. Lozier, R.F. Boisvert, C.W. Clark. NIST Handbook of Mathematical Functions. Cambridge University Press (2010). 951 p.

[30] С.В. Егоров, Л.С. Успенская. Письма в ЖЭТФ 103, 298 (2016). 